\title{
Subspace system identification of support excited structures-part II: gray-box interpretations and damage detection
}

\author{
Junhee Kim and Jerome P. Lynch* \\ Department of Civil and Environmental Engineering, University of Michigan, Ann Arbor, MI, USA
}

\begin{abstract}
SUMMARY
A theoretical framework is presented for the estimation of the physical parameters of a structure (i.e., mass, stiffness, and damping) from measured experimental data (i.e., input-output or output-only data). The framework considers two state-space models: a physics-based model derived from first principles (i.e., white-box model) and a data-driven mathematical model derived by subspace system identification (i.e., black-box model). Observability canonical form conversion is introduced as a powerful means to convert the data-driven mathematical model into a physically interpretable model that is termed a gray-box model. Through an explicit linking of the white-box and gray-box model forms, the physical parameters of the structural system can be extracted from the gray-box model in the form of a finite element discretization. Prior to experimental verification, the framework is numerically verified for a multi-DOF shear building structure. Without a priori knowledge of the structure, mass, stiffness, and damping properties are accurately estimated. Then, experimental verification of the framework is conducted using a six-story steel frame structure under support excitation. With a priori knowledge of the lumped mass matrix, the spatial distribution of structural stiffness and damping is estimated. With an accurate estimation of the physical parameters of the structure, the gray-box model is shown to be capable of providing the basis for damage detection. With the use of the experimental structure, the gray-box model is used to reliably estimate changes in structural stiffness attributed to intentional damage introduced. Copyright (C) 2012 John Wiley \& Sons, Ltd.
\end{abstract}

Received 23 March 2011; Revised 14 February 2012; Accepted 21 February 2012

KEY WORDS: subspace system identification; structural health monitoring; support-excited structure; structural dynamics; physics-based model; data-driven finite element model

\section{INTRODUCTION}

System identification is the art and science of creating an accurate mathematical model of a dynamic system using experimental data [1]. System identification has been under development in a number of different engineering communities since the 1960s. In the control theory community, system identification has been extensively studied since the establishment of the prediction error method by Ljung and his colleagues in the 1980s [2]. For example, an accurate prediction of the behavior of a plant is critical for the design of feedback control algorithms. When modeling the plant by physicsbased principles is difficult, system identification can then be used to provide the control engineer with a rigorous mathematical model that fully describes the plant dynamics. In the civil engineering community, system identification has played a vitally important role in the assessment of the health of a monitored structure [3]. For example, a mathematical model of the monitored structure before and after damage could have notable differences that can be attributed to the damage.

\footnotetext{
*Correspondence to: Jerome P. Lynch, Department of Civil and Environmental Engineering, University of Michigan, 2380 G. G. Brown Building, Ann Arbor, MI, USA.

E-mail: jerlynch@umich.edu
} 
The prediction error method requires a canonical parameterization when applied to state-space model estimation. A nonlinear search is often required to solve the optimization problem posed by the prediction error method; this can lead to poor convergence, especially for multi-input multioutput (MIMO) systems. An early application of the prediction error method to the identification of structures is reported by Hjelmstad et al. [4]. Their study utilized the finite element formulation as the canonical parameterization under the assumption that the geometry of the structure is known a priori. As part of their approach, the nonlinear search is conducted along the time axis to estimate the system parameters. In the 1990s, a new approach to system identification emerged in the control theory community termed subspace system identification [5-7]. The most notable characteristic of subspace system identification is its elimination of the need to specify the canonical parameterization of the mathematical model of the system. Rather, subspace system identification allows one to ignore the underlying physics of the system and to estimate a mathematical model of the system directly from measurement data. Furthermore, subspace system identification does not require nonlinear search methods. While subspace identification has proven to be a very powerful tool to control engineers [1], the civil engineering community has been slow to adopt the method because of the absence of a physical explanation of the method and its complex mathematical derivation. Only recently has subspace system identification made major inroads in the civil engineering field. To further promote subspace identification to the civil engineering community, the companion paper [9] to this paper provides a detailed explanation of the method using geometrical interpretations that relate directly to classical structural dynamics. This study will build on the foundation provided by the first paper to extend subspace identification into the realm of structural health monitoring (SHM).

This paper offers a bridge between the civil engineering and the control systems communities who are engaged in system identification. First, the physics-based models (termed white-box models) popular in the civil engineering community are derived for support-excited structures. Second, entirely data-driven mathematical models (termed black-box models) are identified from experimental data by means of subspace system identification. What might appear to be two different representations of the system can be equated through the use of linear system theory $[10,11]$. Specifically, the mathematical model of the system can be manipulated through canonical form conversion to allow for physical interpretation of the model's final form; such models are herein referred to as gray-box models. The ability to now extract physical parameters from the gray-box model allows subspace system identification methods to be an integral part of future SHM strategies. The overarching strategy proposed in this paper is summarized in Figure 1. For validation, a series of

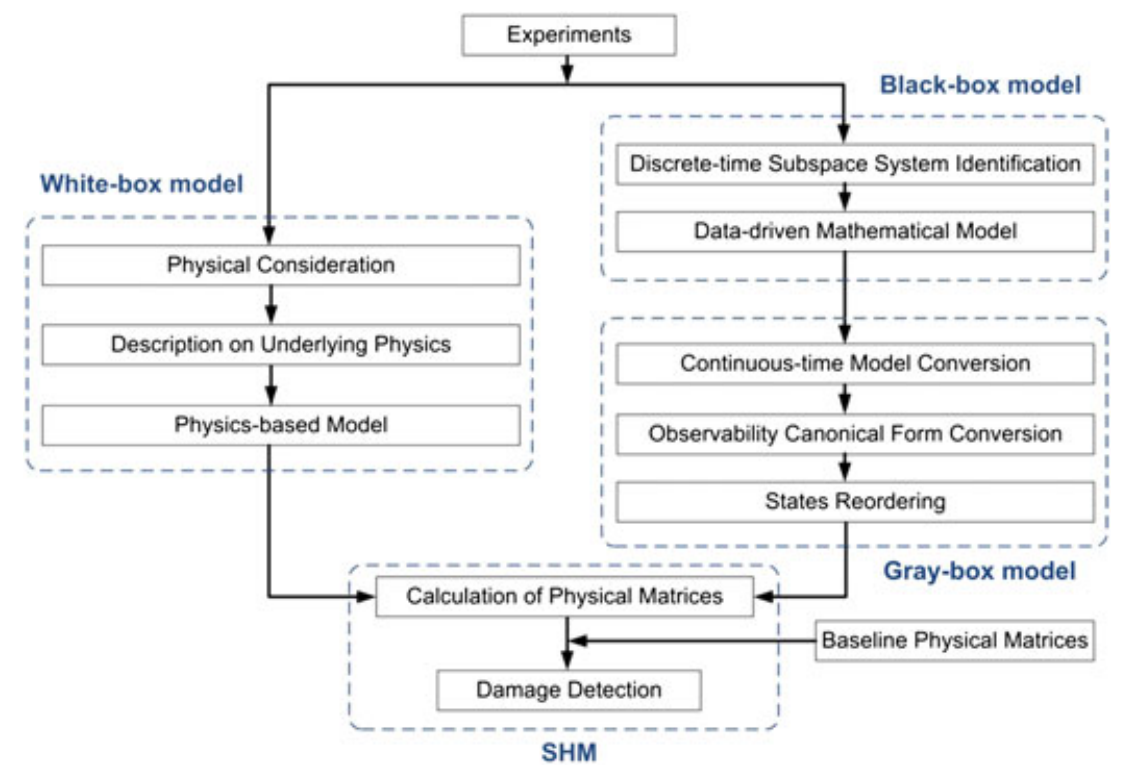

Figure 1. Algorithmic flow of the proposed estimation of physical parameters from gray-box models. 
experimental vibration tests are conducted on the same six-story steel frame structure utilized in the companion paper [9]. A variety of damage cases are introduced in the structure in different locations and in varying severity. Physical parameter estimation is conducted using gray-box models formulated from input-output data and output-only data sets. As will be shown, quantitative evaluation of the physical parameters leads to an accurate means of detecting the location and severity of structural damage.

\section{STATE-SPACE MODEL FORMULATION FROM UNDERLYING PHYSICS}

The equation of motion of a discrete-time finite DOF structural system with $n$ lumped masses and excited by base motion (Figure 2) can be formulated as follows:

$$
\mathbf{M} \ddot{\mathbf{u}}^{t}(t)+\mathbf{F}_{n c}(\dot{\mathbf{u}}(t), \mathbf{u}(t))+\mathbf{K u}(t)=\{\mathbf{0}\}
$$

where $\mathbf{M}=\sum_{e}^{n} \mathbf{M}^{e} \in \mathfrak{l}^{n \times n}, \mathbf{K}=\sum_{e}^{n} \mathbf{K}^{e} \in \mathfrak{i}^{n \times n}$, and $\mathbf{F}_{n c}=\sum_{e}^{n} \mathbf{F}_{n c}{ }^{e} \in \mathbb{C}^{n \times n}$ correspond to the system mass, stiffness, and non-conservative force matrices, respectively. In addition, $\mathbf{u}^{t} \in \mathfrak{h}^{n}$ and $\mathbf{u} \in \mathfrak{i}^{n}$ correspond to the total and relative (with respect to the structure base) displacement vectors, respectively. The first term in Equation (1) represents the inertia force, which is the rate of change of momentum of any mass node. The third term represents the spring force, which is a linear-elastic approximation of the structure based on Hooke's law. The second term represents non-conservative forces. Generally, non-conservative forces are related to the dissipation of energy and are typically considered as damping forces. Numerous damping forces have been formulated in the field of structural dynamics. In this study, viscous damping is adopted because it can be easily modeled as a dashpot whose damping force is linearly proportional to velocity. Using equivalent viscous damping, the equation of motion can be written as follows:

$$
\mathbf{M} \ddot{\mathbf{u}}^{t}(t)+\mathbf{C u}(t)+\mathbf{K u}(t)=\{\mathbf{0}\}
$$

where $\mathbf{C}=\sum_{e}^{n} \mathbf{C}^{e} \in \mathfrak{R}^{n \times n}$ is the system damping matrix. The total displacement in Equation (2) can be expressed as the sum of the support motion $u_{g}(t) \in \mathfrak{l}$ and the relative displacement of the structure, $\mathbf{u}$. Because the relative displacement is parallel to the absolute displacement, the total displacement of the shear structure is given by

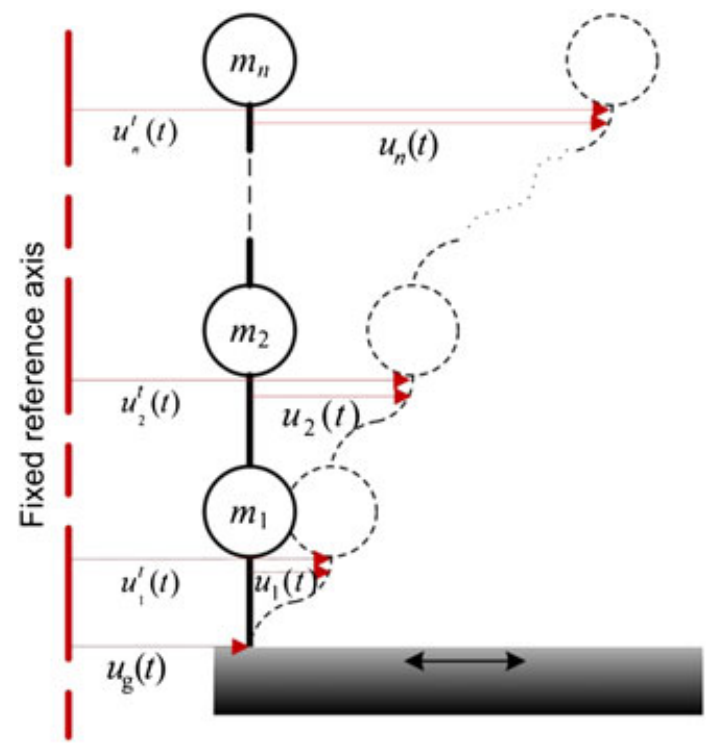

Figure 2. Lumped mass, shear structure deformation under base motion excitation. 


$$
\mathbf{u}^{t}(t)=\mathbf{u}(t)+\{\mathbf{1}\} u_{g}(t)
$$

where $\{\mathbf{1}\} \in \mathfrak{R}^{n}$ is a unitary vector. By substituting Equation (3) in Equation (2), the equation of motion with respect to the ground motion is formulated as follows:

$$
\mathbf{M u}(t)+\mathbf{C u}(t)+\mathbf{K u}(t)=-\mathbf{M}\{\mathbf{1}\} \ddot{u}_{g}(t)
$$

The equation of motion defined by Equation (4) is converted to a form compatible with the data-driven black-box model to be formulated later. Specifically, the state-space model is formulated in a manner that acceleration outputs can be used, yet the state-space model fits into a canonical form. This is in contrast to conventional methods where the state of the whitebox model is defined by the displacement and velocity vectors of the system DOFs regardless of the output measurements. The problem arises when the traditional state (consisting of displacements and velocities) is combined with acceleration outputs; the output matrix C, for this approach would be a full matrix, and the state-space model would not fit into a canonical form. Hence, a rather unorthodox state will be defined on the basis of acceleration and its differential. The differential operator is applied twice to both sides of Equation (4) and mass normalized:

$$
\dddot{\mathbf{u}}(t)=-\mathbf{M}^{-1} \mathbf{K} \ddot{\mathbf{u}}(t)-\mathbf{M}^{-1} \mathbf{C} \ddot{\mathbf{u}}(t)-\{\mathbf{1}\} \dddot{u}_{g}(t)
$$

By defining the following state

$$
\mathbf{x}(t):=\left[\begin{array}{ll}
\ddot{\mathbf{u}}(t)^{T} & \dddot{\mathbf{u}}(t)^{T}
\end{array}\right]^{T}
$$

a state-space model can be defined in the continuous-time domain:

$$
\dot{\mathbf{x}}(t)=\mathbf{A}_{\mathbf{c}} \mathbf{x}(t)+\overline{\mathbf{B}}_{\mathbf{c}} \dddot{u}_{g}(t)
$$

where

$$
\begin{gathered}
\mathbf{A}_{\mathbf{c}}=\left[\begin{array}{cc}
\mathbf{0} & \mathbf{I} \\
-\mathbf{M}^{-1} \mathbf{K} & -\mathbf{M}^{-1} \mathbf{C}
\end{array}\right] \in \mathfrak{\Re}^{2 n \times 2 n} \\
\overline{\mathbf{B}}_{\mathbf{c}}=\left[\begin{array}{c}
\{\mathbf{0}\} \\
-\{\mathbf{1}\}
\end{array}\right] \in R^{2 n}
\end{gathered}
$$

If it is assumed that an accelerometer is installed on every lumped mass, then the observation equation can be formed. Absolute acceleration $\dot{\mathbf{u}}^{t}(t)$, is calculated by applying the differential operator twice to Equation (3) as follows:

$$
\ddot{\mathbf{u}}^{t}(t)=\ddot{\mathbf{u}}(t)+\{\mathbf{1}\} \ddot{u}_{g}(t)
$$

Accelerometers are electromechanical systems, which have very fast dynamics compared to structural system dynamics. Thus, the fundamental dynamics of the sensor can be ignored. Hence, the observation equation does not involve a differential equation. By the predefined state vector, the observation equation is formulated as follows: 


$$
\mathbf{y}(t)=\mathbf{C}_{\mathbf{c}} \mathbf{x}(t)+\mathbf{D}_{\mathbf{c}} \ddot{u}_{g}(t)
$$

where $\mathbf{C}_{\mathbf{c}}=\left[\begin{array}{ll}\mathbf{I} & \mathbf{0}\end{array}\right] \in \mathfrak{\Re}^{n \times 2 n}$ and $\mathbf{D}_{\mathbf{c}}=\{\mathbf{1}\} \in \mathfrak{\Re}^{n}$. The continuous-time state-space model (Equations (7) and (11)) derived from the underlying physics of the system is referred to as the 'white-box model' and schematically pictured in Figure 3(a).

\section{GRAY-BOX MODEL BY OBSERVABILITY CANONICAL FORM CONVERSION}

A state-space model is created using the input-output data set of a support-excited structure and subspace system identification [9]. Let us assume the discrete-time system matrices, $\mathbf{A}_{\mathbf{d}} \in \mathfrak{R}^{2 n \times 2 n}, \mathbf{B}_{\mathbf{d}} \in$ $R^{2 n}, \mathbf{C}_{\mathbf{d}} \in \mathfrak{A i}^{n \times 2 n}$, and $\mathbf{D}_{\mathbf{d}} \in \mathfrak{A i}^{n}$ are identified by the N4SID algorithm using the measured ground acceleration (i.e., input) and the absolute accelerations of the structure masses (i.e., output). Because the identified data-driven model is a black-box model, the states are arbitrary and unknown. There are mathematical procedures that allow the black-box model to be physically interpreted; such conversions render the model a gray-box model. This work will introduce observability canonical form conversion as a computationally efficient means of deriving a physically meaningful gray-box model from a black-box model. The method presented in this paper differs from previous work in gray-box modeling in the civil engineering domain. For example, Xiao et al. [12] proposed a methodology to directly extract physical system matrices from a minimal realization with a priori information of the structure's mass distribution. Although offering a one-step transformation, their canonical form conversion does not directly account for the physical principles of the system through the conversion. Similarly, Lus et al. [13] provides a means of extracting physical properties from black-box state-space models through a transformation that maps the arbitrary state space to one consistent with the symmetric eigenvalue problem from which mass, damping and stiffness properties of the system can be extracted. Their approach requires more steps and is therefore more computationally expensive to implement.

The physics-based model previously developed in Section 2 is defined in the continuous-time domain. Hence, the discrete-time state-space model obtained from susbspace system identification must be converted into the continuous-time domain as follows:

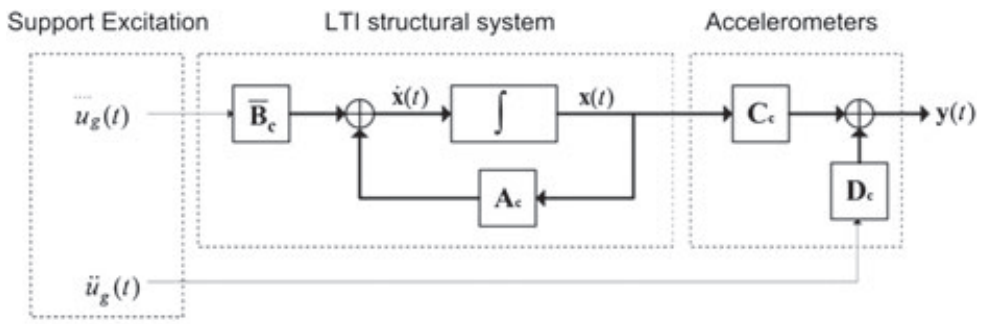

(a)

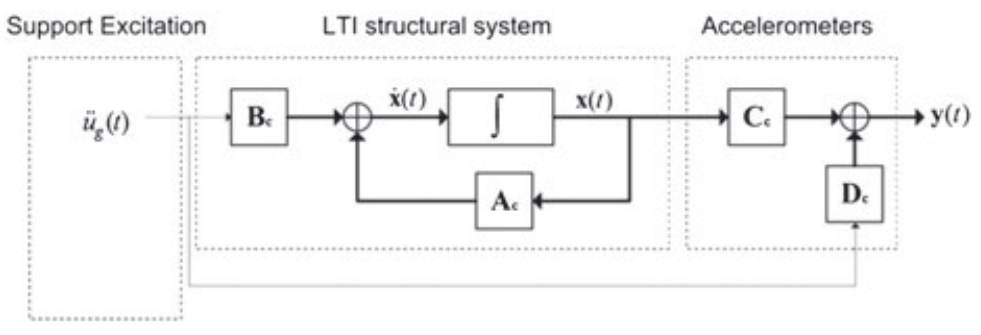

(b)

Figure 3. Continuous-time state-space model for support-excited structures with acceleration output: (a) physics-based model; and (b) data-driven mathematical model. 


$$
\begin{gathered}
\mathbf{A}_{\mathbf{c}}=\frac{1}{\Delta t} \ln \left(\mathbf{A}_{\mathbf{d}}\right) \\
\mathbf{B}_{\mathbf{c}}=\left(\int_{0}^{\Delta t} \exp \left(\mathbf{A}_{\mathbf{c}} \tau\right) d \tau\right)^{-1} \mathbf{B}_{\mathbf{d}} \\
\mathbf{C}_{\mathbf{c}}=\mathbf{C}_{\mathbf{d}} ; \quad \mathbf{D}_{\mathbf{c}}=\mathbf{D}_{\mathbf{d}}
\end{gathered}
$$

where $\Delta t$ is the sampling time step. Continuous-time state-space equations are given with the identified matrices as follows:

$$
\begin{aligned}
\dot{\mathbf{x}}(t) & =\mathbf{A}_{\mathbf{c}} \mathbf{x}(t)+\mathbf{B}_{\mathbf{c}} \ddot{u}_{g}(t) \\
\mathbf{y}(t) & =\mathbf{C}_{\mathbf{c}} \mathbf{x}(t)+\mathbf{D}_{\mathbf{c}} \ddot{u}_{g}(t)
\end{aligned}
$$

The continuous-time state-space mathematical model is depicted in Figure 3(b). The model relates the system input $\ddot{u}_{g}(t)$, with the system output $\mathbf{y}(t)$, using an internal intermediate state $\mathbf{x}(t)$. The output is the absolute acceleration measured by accelerometers. With the application of a coordinate transformation to the intermediate state, it is possible to define the state with specific physical parameters (i.e., acceleration) such as those defined in the system output. For example, the sensor system matrix $\mathbf{C}_{\mathbf{c}}$, would be the identity matrix for the measured states if the defined state was identical to the measured absolute acceleration of the structure. This is the concept of the observability canonical form of the state-space model realization [10]. As will be described, the observability canonical form conversion will be an important step in the creation of a gray-box model from the black-box model.

If $\left(\mathbf{A}_{\mathbf{c}}, \mathbf{B}_{\mathbf{c}}, \mathbf{C}_{\mathbf{c}}, \mathbf{D}_{\mathbf{c}}\right)$ is any minimal realization of a true system, then $\left(\mathbf{A}_{\mathbf{c}}, \mathbf{B}_{\mathbf{c}}, \mathbf{C}_{\mathbf{c}}, \mathbf{D}_{\mathbf{c}}\right)$ and $\left(\mathbf{A}_{\mathbf{c}}^{\prime}, \mathbf{B}_{\mathbf{c}}^{\prime}, \mathbf{C}_{\mathbf{c}}^{\prime}, \mathbf{D}^{\prime}{ }_{\mathbf{c}}\right)$ can be related to one another through the nonsingular transformation matrix $\mathbf{T}$ :

$$
\mathbf{A}_{\mathbf{c}}^{\prime}=\mathbf{T} \quad \mathbf{A}_{\mathbf{c}} \mathbf{T}^{-1} ; \quad \mathbf{B}_{\mathbf{c}}^{\prime}=\mathbf{T B}_{\mathbf{c}} ; \quad \mathbf{C}_{\mathbf{c}}^{\prime}=\mathbf{C}_{\mathbf{c}} \mathbf{T}^{-1} ; \quad \mathbf{D}_{\mathbf{c}}^{\prime}=\mathbf{D}_{\mathbf{c}}
$$

The observability canonical form can be constructed by taking the transformation matrix $\mathbf{T}$, to be the observability matrix $\mathcal{O}$ (i.e., $\mathbf{T}=\mathcal{O}$ ). The observation matrix $\mathbf{C}_{\mathbf{c}}$ in Equation (16) can be expressed with the $n$ rows as follows:

$$
\mathbf{C}_{\mathbf{c}}=\left[\begin{array}{c}
\mathbf{c}_{1} \\
\vdots \\
\mathbf{c}_{n}
\end{array}\right]
$$

where $\mathbf{c}_{1}, \cdots, \mathbf{c}_{n} \in R^{1 \times 2 n}$ are $n$ row vectors. With the use of row-wise expressions of $\mathbf{C}_{\mathbf{c}}$, the observability matrix can be composed as follows:

$$
\mathcal{O}=\left[\begin{array}{llllllllll}
\mathbf{c}_{1}^{T} & \cdots & \left(\mathbf{c}_{1} \mathbf{A}_{\mathbf{c}}^{\gamma_{1}-1}\right)^{T} & \mathbf{c}_{2}^{T} & \cdots & \left(\mathbf{c}_{2} \mathbf{A}_{\mathbf{c}}^{\gamma_{2}-1}\right)^{T} & \cdots & \mathbf{c}_{n}^{T} & \cdots & \left(\mathbf{c}_{n} \mathbf{A}_{\mathbf{c}}^{\gamma_{n}-1}\right)^{T}
\end{array}\right]^{T}
$$

where $\gamma_{i}(i=1,2, \cdots, n)$ are observability indices. Since a minimal state-space realization is completely observable, the rank of the observability matrix must be $2 n$. As a result, all observability indices $\gamma_{i}$, in Equation (19) are equal to 2. By Equation (17), the transformed system matrices using the observability matrix yields the following structure: 


$$
\mathbf{A}_{\mathbf{c}}=\left[\begin{array}{cc|cc|cc|cc}
0 & 1 & 0 & 0 & \ldots & \ldots & 0 & 0 \\
* & * & * & * & \ldots & \ldots & * & * \\
\hline 0 & 0 & 0 & 1 & \ldots & \ldots & 0 & 0 \\
* & * & * & * & \ldots & \ldots & * & * \\
\hline \vdots & \vdots & \vdots & \vdots & \ddots & & \vdots & \vdots \\
\vdots & \vdots & \vdots & \vdots & & \ddots & \vdots & \vdots \\
\hline 0 & 0 & 0 & 0 & \ldots & \ldots & 0 & 1 \\
* & * & * & * & \ldots & \ldots & * & *
\end{array}\right]
$$

$$
\mathbf{C}_{\mathbf{c}}=\left[\begin{array}{cc|cc|cc|cc}
1 & 0 & 0 & 0 & \cdots & \cdots & 0 & 0 \\
\hline 0 & 0 & 1 & 0 & \cdots & \cdots & 0 & 0 \\
\hline \vdots & \vdots & \vdots & \vdots & \ddots & & \vdots & \vdots \\
\vdots & \vdots & \vdots & \vdots & & \ddots & \vdots & \vdots \\
\hline 0 & 0 & 0 & 0 & \cdots & \cdots & 0 & 0 \\
\hline 0 & 0 & 0 & 0 & \cdots & \cdots & 1 & 0
\end{array}\right]
$$

where * corresponds to non-zero entities. The order of the states in the experimentally identified model is different from that of the predefined states in the physics-based model. By reordering the state as $\mathbf{x}(t)=\left[\begin{array}{llllll}\ddot{u}_{1}(t) & \cdots & \ddot{u}_{n}(t) & \dddot{u}_{1}(t) & \cdots & \dddot{u}_{n}(t)\end{array}\right]^{T}$, the identified system matrices $\left(\mathbf{A}_{\mathbf{c}}, \mathbf{B}_{\mathbf{c}}, \mathbf{C}_{\mathbf{c}}, \mathbf{D}_{\mathbf{c}}\right)$ will conveniently have the following form:

$$
\begin{aligned}
& \mathbf{A}_{\mathbf{c}}=\left[\begin{array}{cc}
\mathbf{0} & \mathbf{I} \\
\mathbf{X} & \mathbf{Y}
\end{array}\right] \in \mathfrak{R}^{2 n \times 2 n} \\
& \mathbf{B}_{\mathbf{c}}=\left[\begin{array}{c}
\{\mathbf{\approx} \mathbf{0}\} \\
\{\mathbf{Z}\}
\end{array}\right] \in \mathfrak{l}^{2 n} \\
& \mathbf{C}_{\mathbf{c}}=\left[\begin{array}{ll}
\mathbf{I} & \mathbf{0}
\end{array}\right] \in \mathfrak{\Re}^{n \times 2 n} \\
& \mathbf{D}_{\mathbf{c}}=\{\mathbf{W}\} \in \mathfrak{\Re}^{n}
\end{aligned}
$$

where $\{\approx \mathbf{0}\} \in \mathfrak{i}^{n}$ is a column vector with non-zero but trivial values and $\mathbf{X} \in \mathfrak{i}^{n \times n}, \mathbf{Y} \in \mathfrak{i}^{n \times n},\{\mathbf{Z}\} \in \mathfrak{i}^{n}$, and $\{\mathbf{W}\}$ are portions of the system matrices that have non-zero values. By the process of observability canonical form conversion and state reordering, the continuous-time state-space mathematical model derived from subspace system identification has now been converted into a gray-box model whose internal system description can now be physically mapped to the white-box model.

\section{PHYSICAL PARAMETER ESTIMATION USING THE GRAY-BOX MODEL}

\subsection{Methodology}

Because the system properties are fully captured in the system matrices $\left\{\mathbf{A}_{\mathbf{c}}, \mathbf{B}_{\mathbf{c}}\right\}$, the physical parameters of the structural system can be estimated through a direct comparison of Equations (8) 
and (9) of the physics-based model (i.e., white-box model) and Equations (22) and (23) of the data-driven mathematical model (i.e., gray-box model). This approach is depicted in Figure 4(a). However, as seen in Figure 4(a), a gap still exists between the two models because of model discrepancies. The existence of this gap was also an issue in the control theory community when considering both prediction error and subspace system identification methods as depicted in Figure 4(b). This abstract gap problem was solved by Ljung, Åström, and Söderström in the mid 1980s and is often referred to as the 'Ljung's cleanup' [2]. By making a clear distinction between the true system and the model set [1], system identification must be considered as an approximation problem (i.e., minimize the gap) as opposed to an exact problem (i.e., close the gap). Hence, system identification is fundamentally a problem of defining a model with an acceptable quality for an intended application. In this study, model quality is assessed in the context of physical parameter estimation for damage detection. As seen in Figure 3, the two models (i.e., physics-based and data-driven mathematical models) are similar but not identical; the system input matrices $\overline{\mathbf{B}}_{\mathbf{c}}$ and $\mathbf{B}_{\mathbf{c}}$ have different physical meaning, especially when making absolute acceleration measurements of support-excited structures. Admitting this difference, this study aims to estimate the physical parameters of the system by comparing the system matrices $\left(\mathbf{A}_{\mathbf{c}}\right)$ of both models (i.e., white-box and gray-box models). It should be noted that this strategy could also be equivalently applied to models derived from output-only system identification.

Comparing the system matrix $\mathbf{A}_{\mathbf{c}}$, of the white-box and gray-box models, the following relationships are obtained:

$$
\begin{aligned}
& \mathbf{X}=-\mathbf{M}^{-1} \mathbf{K} \\
& \mathbf{Y}=-\mathbf{M}^{-1} \mathbf{C}
\end{aligned}
$$

Pre-multiplying $\mathbf{M}$ on both sides of Equations (26) and (27) yields

$$
\begin{aligned}
& \mathbf{M X}=-\mathbf{K} \\
& \mathbf{M Y}=-\mathbf{C}
\end{aligned}
$$

Numerous solutions for $\mathbf{M}, \mathbf{C}$, and $\mathbf{K}$ given the gray-box $\mathbf{A}_{\mathbf{c}}$ sub-matrices $\mathbf{X}$ and $\mathbf{Y}$ exist. For the purpose of damage detection through system identification, the structure's spatial distribution of stiffness and damping are more relevant because structural damage is viewed as a physical phenomenon that is closely related to structural stiffness and damping rather than mass. Thus, damage is often quantified as a

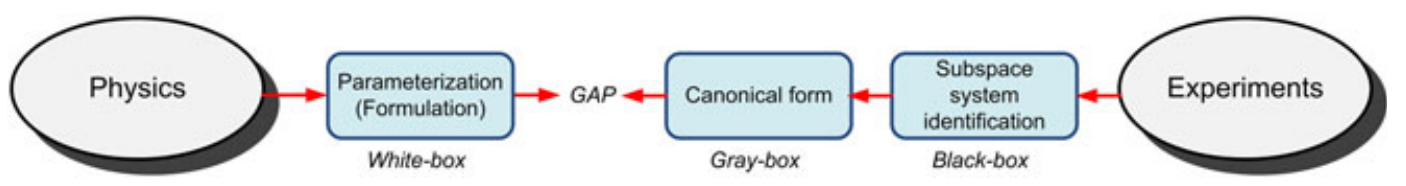

(a)

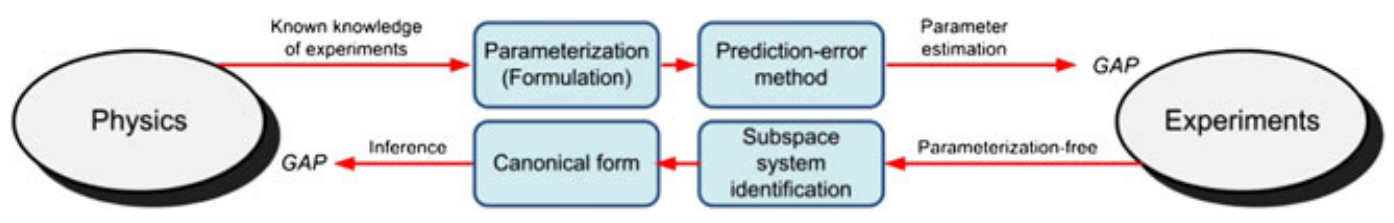

(b)

Figure 4. System identification of a dynamic system: (a) the approach proposed in this study; and (b) two common approaches taken in the control community. 
change in the identified $\mathbf{K}$ and $\mathbf{C}$ under the assumption of an invariant distribution of mass. In this study, a priori knowledge on the mass distribution of a structure is utilized for the purpose of extracting $\mathbf{K}$ and $\mathbf{C}$. However, considering the potential difficulty of accurately acquiring the full knowledge of a structure's mass, only the case of partial a priori knowledge of the mass distribution is considered.

If the mass distribution of the structure is accurately known (i.e., $\mathbf{M}$ can be determined), then by Equations (28) and (29), $\mathbf{K}$ and $\mathbf{C}$ can be easily calculated. However, if $\mathbf{M}$ is not known completely, the problem is a little bit more challenging. This problem of finding matrices can be effectively formulated using the concept of vectorized expressions [14, 15]:

$$
\begin{aligned}
& \operatorname{vec}(\mathbf{M X})=-\operatorname{vec}(\mathbf{K}) \in \mathfrak{\Re}^{n^{2}} \\
& \operatorname{vec}(\mathbf{M Y})=-\operatorname{vec}(\mathbf{C}) \in \mathfrak{\Re}^{n^{2}}
\end{aligned}
$$

where $\operatorname{vec}(\cdot)$ is the vectorized expression of the matrix; in other words, the matrix is stacked into a single column vector. Considering the Kronecker product $\otimes$, it can be stated that

$$
\begin{aligned}
& \operatorname{vec}(\mathbf{M X})=\left[\mathbf{X}^{T} \otimes \mathbf{I}\right] \operatorname{vec}(\mathbf{M})=-\operatorname{vec}(\mathbf{K}) \\
& \operatorname{vec}(\mathbf{M Y})=\left[\mathbf{Y}^{T} \otimes \mathbf{I}\right] \operatorname{vec}(\mathbf{M})=-\operatorname{vec}(\mathbf{C})
\end{aligned}
$$

where $\left[\mathbf{X}^{T} \otimes \mathbf{I}\right] \in \mathfrak{1}^{n^{2} \times n^{2}}$ and $\left[\mathbf{Y}^{T} \otimes \mathbf{I}\right] \in \mathfrak{k}^{n^{2} \times n^{2}}$. With the reciprocal theorem for linear structural systems, $\mathbf{K}$ and $\mathbf{C}$ are symmetric (i.e., $\mathbf{K}=\mathbf{K}^{T}$ and $\mathbf{C}=\mathbf{C}^{T}$ ). Applying this fact to Equations (28) and (29) yields

$$
\begin{aligned}
& \mathbf{M X}=\mathbf{X}^{T} \mathbf{M}=\mathbf{K} \\
& \mathbf{M Y}=\mathbf{Y}^{T} \mathbf{M}=\mathbf{C}
\end{aligned}
$$

From Equations (34) and (35), the following Kronecker product relationships are obtained

$$
\begin{aligned}
& {\left[\mathbf{X}^{T} \otimes \mathbf{I}\right] \operatorname{vec}(\mathbf{M})=\left[\mathbf{I} \otimes \mathbf{X}^{T}\right] \operatorname{vec}(\mathbf{M})} \\
& {\left[\mathbf{Y}^{T} \otimes \mathbf{I}\right] \operatorname{vec}(\mathbf{M})=\left[\mathbf{I} \otimes \mathbf{Y}^{T}\right] \operatorname{vec}(\mathbf{M})}
\end{aligned}
$$

By combining Equations (32), (33), (36), and (37), the form of a linear regression can be composed as follows:

$$
\left[\begin{array}{ccc}
{\left[\mathbf{X}^{T} \otimes \mathbf{I}\right.} & \mathbf{0} & {[\mathbf{I}]} \\
{\left[\mathbf{Y}^{T} \otimes \mathbf{I}\right.} & {[\mathbf{I}]} & \mathbf{0} \\
{\left[\mathbf{X}^{T} \otimes \mathbf{I}\right]-\left[\mathbf{I} \otimes \mathbf{X}^{T}\right]} & \mathbf{0} & \mathbf{0} \\
{\left[\mathbf{Y}^{T} \otimes \mathbf{I}\right]-\left[\mathbf{I} \otimes \mathbf{Y}^{T}\right]} & \mathbf{0} & \mathbf{0}
\end{array}\right]\left\{\begin{array}{l}
\operatorname{vec}(\mathbf{M}) \\
\operatorname{vec}(\mathbf{C}) \\
\operatorname{vec}(\mathbf{K})
\end{array}\right\}=\{\mathbf{0}\}
$$

Equation (38) can be symbolized as $\mathbf{P q}=\{\mathbf{0}\}$ where $\mathbf{P} \in \mathfrak{\Re}^{4 n^{2} \times 3 n^{2}}, \mathbf{q} \in \mathfrak{\Re}^{3 n^{2}}$, and $\{\mathbf{0}\} \in \mathfrak{h}^{4 n^{2}}$. Depending on the partial a priori knowledge on mass $\mathbf{P}$ and $\mathbf{q}$ can be partitioned by pivoting as follows:

$$
\left[\begin{array}{ll}
\mathbf{P}_{1} & \mathbf{P}_{2}
\end{array}\right]\left\{\begin{array}{c}
\mathbf{q}_{\text {known }} \\
\mathbf{q}_{\text {TBD }}
\end{array}\right\}=\{\mathbf{0}\}
$$

where $\mathbf{q}_{\text {known }}$ reflects known mass parameters and $\mathbf{P}_{\mathbf{1}}$ corresponds to a regressor matrix; $\mathbf{q}_{\mathbf{T B D}}$ is the to-be-determined (TBD) mass, stiffness, and damping parameters, and $\mathbf{P}_{2}$ corresponds to another 
regressor matrix. The least square solution for the unknown parameter vector $\mathbf{q}_{\mathbf{T B D}}$, can be calculated from the Moore-Penrose pseudo-inverse as follows:

$$
\mathbf{q}_{\mathbf{T B D}}=\mathbf{P}_{2}^{\dagger}\left(-\mathbf{P}_{1} \mathbf{q}_{\text {known }}\right)
$$

The regression problem formulated in Equation (39) is quite powerful in its generality. Since the right-hand side of the equation is the null space, its rank deficiency is 1 . As such, it suggests that only one known model parameter is needed to solve for the other unknown (i.e., TBD) parameters. In the presence of noise, making assumptions about more than one parameter is prudent since the estimation of the unknown parameters $\left(\mathbf{q}_{\mathbf{T B D}}\right)$ by Equation (40) will yield more accurate results as the size of $\mathbf{q}_{\text {known }}$ grows. It should be kept in mind that $\mathbf{q}_{\mathbf{k n o w n}}$ can be populated by mass parameters, stiffness parameters, damping parameters, or a combination of the three. However, physical changes of stiffness and damping is more meaningful in the damage detection problem than changes in mass. Thus, the use of a priori knowledge on structural mass is suggested in this study.

\subsection{Numerical example}

A numerical example is studied before applying the gray-box system identification method to a more complex experimental problem. A three-story lumped mass shear structure excited at each story by a measured force $f$, is simulated (Figure 5(a)). The measured response of the structure is the displacement of each story of the shear structure. Because the example has an identical white-box and gray-box model (Figure 5(b)), the problem is well suited for numerical simulation. Furthermore, the absence of the gap in Figure 4(a) allows performance of the physical parameter estimation to be assessed through numerical simulation. The equation of motion of the multi-story building excited at each story is formulated as follows:

$$
\mathbf{M} \ddot{\mathbf{u}}(t)+\mathbf{C} \dot{\mathbf{u}}(t)+\mathbf{K} \mathbf{u}(t)=\mathbf{f}(t)
$$

By defining the state vector as $\mathbf{x}(t):=\left[\begin{array}{ll}\mathbf{u}(t)^{T} & \dot{\mathbf{u}}(t)^{T}\end{array}\right]^{T}$, the physics-based model is derived as a continuous-time state-space model with system matrices:

$$
\begin{gathered}
\mathbf{A}_{\mathbf{c}}=\left[\begin{array}{cc}
\mathbf{0} & \mathbf{I} \\
-\mathbf{M}^{-1} \mathbf{K} & -\mathbf{M}^{-1} \mathbf{C}
\end{array}\right] \in \mathfrak{\Re}^{2 n \times 2 n} \\
\mathbf{B}_{\mathbf{c}}=\left[\begin{array}{c}
\mathbf{0} \\
\mathbf{M}^{-1}
\end{array}\right] \in \mathfrak{\Re}^{2 n \times n} \\
\mathbf{C}_{\mathbf{c}}=\left[\begin{array}{ll}
\mathbf{I} & \mathbf{0}
\end{array}\right] \in \mathfrak{R}^{n \times 2 n}
\end{gathered}
$$

where $\mathbf{M}^{-1}$ is the inverse of the lumped mass matrix defined as

$$
\mathbf{M}^{-1}=\left[\begin{array}{cccc}
1 / m_{1} & 0 & \cdots & 0 \\
0 & 1 / m_{2} & \cdots & 0 \\
\vdots & \vdots & \ddots & \vdots \\
0 & 0 & \cdots & 1 / m_{n}
\end{array}\right] \in \Re^{n \times n}
$$

Using simulated input-output data, a data-driven mathematical model is derived by the subspace identification method (i.e., N4SID). The resulting black-box model is converted to its gray-box form via the observability canonical form transformation: 


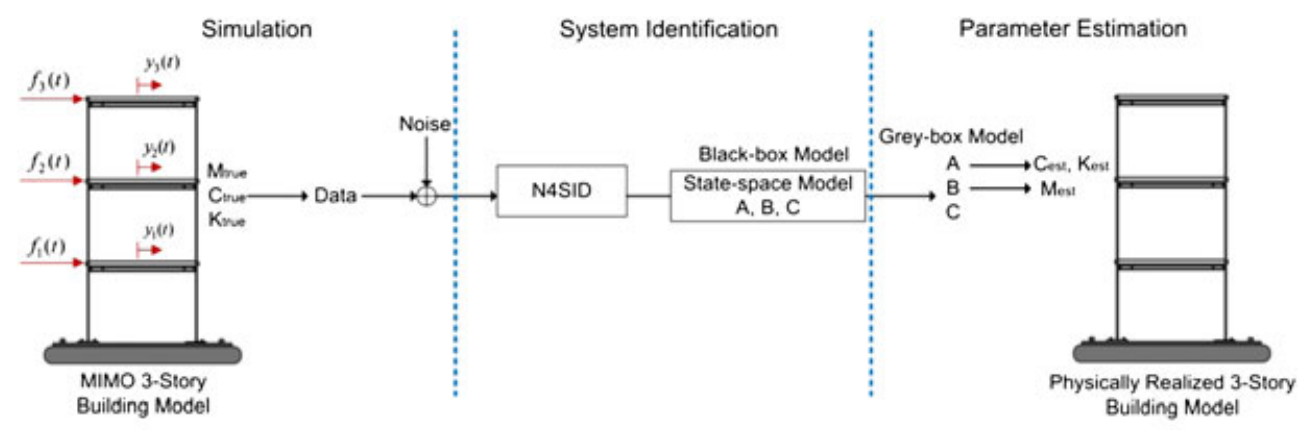

(a)

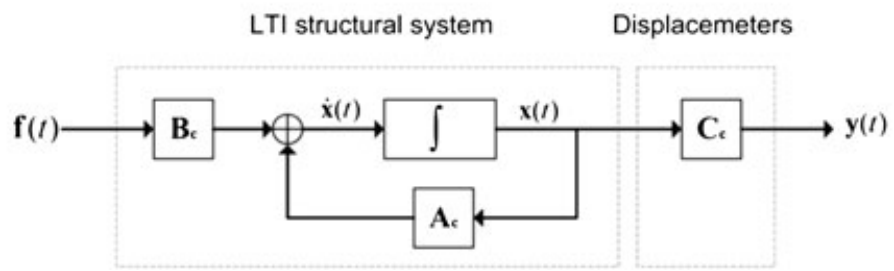

(b)

Figure 5. Numerical example of a three-story lumped mass, shear structure: (a) numerical procedure from simulation to structural parameter estimation; and (b) identical white-box and gray-box models.

$$
\begin{gathered}
\mathbf{A}_{\mathbf{c}}=\left[\begin{array}{cc}
\mathbf{0} & \mathbf{I} \\
\mathbf{X} & \mathbf{Y}
\end{array}\right] \in \mathfrak{l}^{2 n \times 2 n} \\
\mathbf{B}_{\mathbf{c}}=\left[\begin{array}{c}
\approx \mathbf{0} \\
\mathbf{Z}
\end{array}\right] \in \mathfrak{R}^{2 n \times n} \\
\mathbf{C}_{\mathbf{c}}=\left[\begin{array}{ll}
\mathbf{I} & \mathbf{0}
\end{array}\right] \in \mathfrak{A}^{n \times 2 n}
\end{gathered}
$$

where $\approx \mathbf{0} \in R^{n \times n}$ is a matrix composed of near-zero values. By comparison of $\mathbf{B}_{\mathbf{c}}$ in Equations (43) and (47), the mass matrix can be determined. Then, the stiffness and damping matrices are calculated by the comparison of $\mathbf{A}_{\mathbf{c}}$ (i.e., Equations (42) and (46)) with the known mass matrix.

The whole procedure consists of four major steps as illustrated in Figure 5(a): (i) physics-based system matrices are composed and numerical simulation of the system is conducted to generate a complete set of input-output data; (ii) noise is added to the simulated data prior to system identification to simulate realistic sensor readings; (iii) system identification by N4SID is conducted on the input-output data to produce the black-box model of the system; and (iv) gray-box model conversion is implemented to estimate physical parameters from the black-box model. For the physical model, identical inter-story stiffness values are used $\left(1.9 \times 10^{6} \mathrm{~N} / \mathrm{m}\right)$ for every story whereas three different masses are used $\left(m_{1}=800 \mathrm{~kg}, m_{2}=850 \mathrm{~kg}\right.$, and $\left.m_{3}=900 \mathrm{~kg}\right)$. The inter-story damping is assumed to be proportional to the inter-story stiffness (various values will be considered). White noise forces are applied to each floor mass. The displacements of the floors are considered as the system measured output. The sampling rate for the discrete time data is $100 \mathrm{~Hz}$.

Convergence of the model is affected by three major factors: (1) the size of the data set (as the number of data points goes to infinity, the model converges asymptotically under the assumption of a Gaussian stochastic process); (2) noise (white noise contamination of the measured output deteriorates the convergence); and (3) damping components in the system (the influence of small damping can be hidden within the noise content of the output or the output of a system with large damping might not contain enough dynamic behavior for accurate system identification). 
A parametric study is conducted to evaluate the effect of these three factors. However, some challenges do exist when performing the parametric study. First, countless combinations of the three factors exist. Second, a statistical analysis should be conducted; this requires numerous simulations to be implemented for each combination. Third, the simulation study is heavily dependent on the performance of the random number generator used to generate the white noise forcing and noise functions. Thus, it is challenging to perform a precise quantitative analysis of the results. Rather, the overall trend of the effect of the three factors on the system identification results will be explored.

Numerical simulation of the three-story structure is adopted with five different data set lengths considered: 3000, 10,000, 50,000, 100,000, and 500,000 points. Also, five noise ratios are considered on the system output: $0.0,0.001,0.005,0.01$, and 0.03 (RMS). Finally, five damping to stiffness ratios are considered: $0.0,0.0001,0.001,0.005$, and 0.01 . Within the simulation study, the model quality is explored under three different variations in the implementation of the gray-box system identification method: (i) model quality as a function of data length with the noise ratio (0.01) and damping to stiffness ratio (0.0001) held constant; (ii) model quality as a function of sensor noise with the data length (50,000 points) and damping to stiffness ratio (0.0001) held constant; and (iii) model quality as a function of the damping to stiffness ratio with the data length (50,000 points) and sensor noise (0.01 RMS) held constant. To provide a statistical basis for the observations made, 100 numerical simulations are performed under different white noise loading conditions (based on independent random number generators). To assess the model quality, the mass, damping and stiffness parameters of the structure are estimated from the simulated data set. For example, the error associated with the estimated mass parameter is defined as follows:

$$
\mathrm{M}_{\mathrm{e}}(\%)=\frac{1}{n^{2}}\left(\sum_{i, j}^{n}\left|\frac{m_{i j}^{\text {true }}-m_{i j}^{\mathrm{est}}}{m_{i j}^{\mathrm{true}}}\right|\right) \times 100
$$

where $m_{i j}^{\text {true }}$ and $m_{i j}^{\text {est }}$ are the entities of the mass matrix corresponding to the true and estimated models, respectively. In a similar fashion, the stiffness $\mathrm{K}_{\mathrm{e}}(\%)$, and damping $\mathrm{C}_{\mathrm{e}}(\%)$, parameter errors are also calculated.

The results of the numerical parametric study are summarized in a tabulated form in Table I. Mean estimation errors on the system mass and stiffness are very small. The accuracy of the physical parameters of the model increases in tandem with an increase in the number of data points used. As the noise in the sensor output rises, so does the percent error associated with the estimation of mass, damping and stiffness; damping appears to be the most sensitive parameter to sensor noise as would be expected. As the amount of damping in the structure is increased, the estimated damping parameters are more accurate. These results prove that the proposed parameter extraction methodology based on the gray-box model provides accurate results that are sufficient for use in structural health monitoring strategies.

\section{EXPERIMENTAL VERIFICATION}

\subsection{Testbed structure}

The single-bay steel frame structure (Figure 6) previously described in the companion paper [9] to this paper is again adopted for experimental validation of the proposed gray-box system identification. The test structure is a six-story single-bay steel frame building with $1.0-\mathrm{m}$ story heights. Each floor is constructed as a rigid diaphragm $(1.0 \mathrm{~m} \times 1.5 \mathrm{~m} \times 2 \mathrm{~cm}$ thick $)$ welded along all four edges to $5.0 \times 0.5 \mathrm{~cm}$ rectangular beams. Each floor is supported by four steel rectangular $(15.0 \times 2.5 \mathrm{~cm})$ plates acting as columns. The structural parameters of the test structure are summarized in Table II. As shown in Figure 6, the structure is mounted to a shaking table located at the National Center for Research in Earthquake Engineering (NCREE), Taipei, Taiwan; the table is capable of applying six DOFs base motion to excite the structure.

In this study, the support excitation is white noise with a peak ground acceleration of $0.061 \mathrm{~g}$ applied in one direction. Specifically, this motion is applied along the planar weak axis of the structure. Seven 


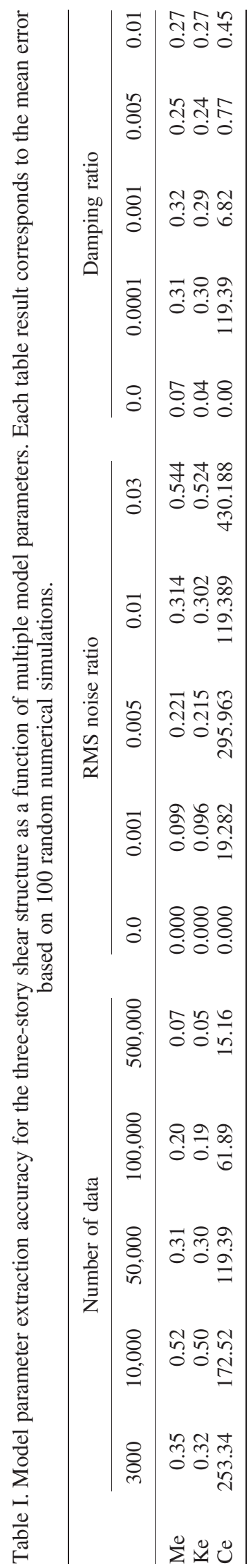


accelerometers are installed to measure the acceleration of the ground motion and the lateral response of each story. A total of seven tests are conducted on the structure. First, the undamaged structure (i.e., baseline) is excited and its response recorded. The next six tests correspond to the damaged structure. Damage is emulated by cutting two of the structural columns below the floor connections at select floor locations. Figure 6(c) shows the cut locations while Table III documents the floors damaged and the level of damage severity for the six damage cases. It should be noted that the emulated damage scenarios are not intended to represent severe damage, but rather minor structural damage possible during a seismic event.

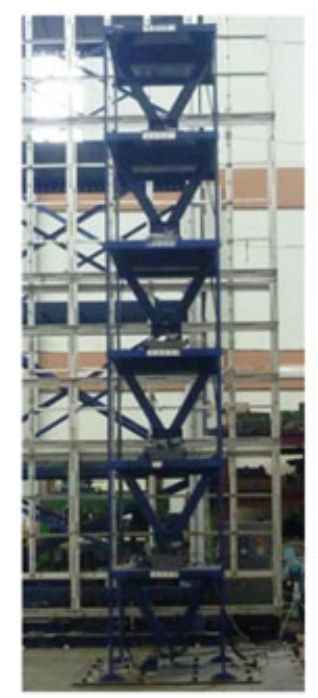

(a)

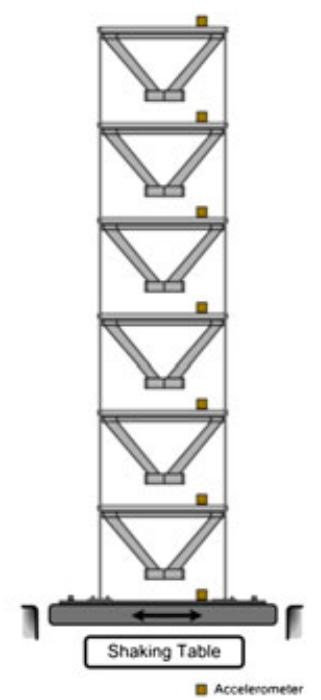

(b)

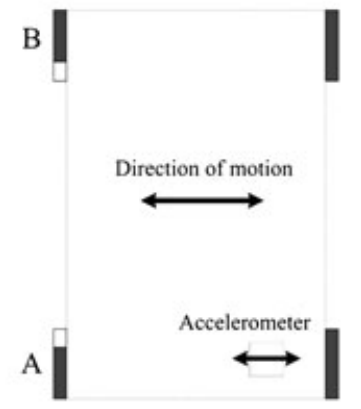

(c)

Figure 6. Testbed structure: (a) six-story single-bay steel building mounted on NCREE shake table; (b) planar schematics with accelerometer locations denoted; and (c) plan view of the structure showing rectangular columns and the introduction of cut damage in columns A and B.

Table II. Theoretical structural parameters of the experimental six-story frame structure.

\begin{tabular}{lc}
\hline Parameter & Value \\
\hline Floor mass & $862 \mathrm{~kg}$ \\
Floor area & $1 \times 1.5 \mathrm{~m}^{2}$ \\
Inter-story height & $1 \mathrm{~m}$ \\
Column cross section & $15 \times 2.5 \mathrm{~cm}^{2}$ \\
Inter-story stiffness & $1.9 \times 10^{6} \mathrm{~N} / \mathrm{m}$
\end{tabular}

Inter-story stiffness is calculated from Euler-Bernoulli beam equations with fixed-fixed end conditions.

Table III. Damage scenarios for the testbed structure.

\begin{tabular}{lcc}
\hline & & Location \\
\cline { 2 - 3 } Case & Under first-floor mass & Under second-floor mass \\
\hline Case 1 & $\mathrm{~A}(6)$ & - \\
Case 2 & $\mathrm{~A}(6), \mathrm{B}(3)$ & - \\
Case 3 & $\mathrm{~A}(6), \mathrm{B}(6)$ & - \\
Case 4 & $\mathrm{~A}(9), \mathrm{B}(9)$ & $\mathrm{A}(6)$ \\
Case 5 & $\mathrm{A}(9), \mathrm{B}(9)$ & $\mathrm{A}(6), \mathrm{B}(6)$ \\
Case 6 & $\mathrm{~A}(9), \mathrm{B}(9)$ & \\
\hline
\end{tabular}

The number in parenthesis denotes induced cut lengths in centimeters. 


\subsection{Identification of the undamaged baseline system}

Estimation of the structural parameters from the experimental data is conducted off-line using 30-s-long data sets (i.e., 3000 data points). First, input-output and output-only subspace system identification is conducted separately to determine optimal state-space black-box models. Through observability canonical form conversion, the gray-box models of the structure are determined. With the a priori knowledge of the structure's distribution of mass (i.e., $862 \mathrm{~kg}$ at each floor), the spatial distribution of structural stiffness and damping are calculated by Equations (28) and (29). For example, for the inputoutput analysis, the stiffness and damping matrices estimated for the baseline structure are as follows:

$$
\hat{\mathbf{K}}=\left[\begin{array}{rrrrrr}
3,260,436 & -1,964,009 & 414,255 & -104,393 & 88,381 & -67,298 \\
-1,788,583 & 3,032,469 & -1,939,778 & 407,177 & 82,730 & -99,864 \\
439,303 & -1,827,917 & 2,932,826 & -1,892,131 & 534,577 & -131,916 \\
-145,435 & 507,923 & -2,002,127 & 3,187,329 & -1,870,920 & 299,167 \\
-33,299 & -73,415 & 440,842 & -1,907,798 & 2,969,031 & -1,462,394 \\
5980 & -116,316 & 130,940 & 298,632 & -1,645,762 & 134,3867
\end{array}\right]
$$

$$
\hat{\mathbf{C}}=\left[\begin{array}{rrlrrr}
1154 & -486 & 1157 & -625 & -59 & 200 \\
79 & -192 & 1659 & -995 & -917 & 555 \\
-398 & -292 & 1264 & -192 & -1721 & 195 \\
-429 & 175 & -610 & 783 & -1373 & -174 \\
-2 & 146 & -819 & 177 & 693 & -1088 \\
923 & -109 & -596 & 294 & 2469 & 832
\end{array}\right]
$$

while for output-only analysis, the stiffness and damping matrices are estimated as follows:

$$
\hat{\mathbf{K}}=\left[\begin{array}{rrrrrr}
3,265,841 & -1,964,712 & 420,615 & -85,008 & 83,801 & -76,696 \\
1,766,359 & 3,027,363 & -1,971,765 & 385,487 & 80,252 & -82,615 \\
478,759 & -1,863,302 & 3,007,121 & -1,903,873 & 526,180 & -144,488 \\
132,824 & 475,307 & -2,015,466 & 3,200,151 & -1,845,411 & 300,643 \\
-47,256 & -41,951 & 430,675 & -1,944,562 & 3,005,266 & -1,544,925 \\
-27,761 & -54,718 & 62,227 & 330,254 & -1,619,674 & 1,378,577
\end{array}\right]
$$

$$
\hat{\mathbf{C}}=\left[\begin{array}{rrrrrr}
2180 & 195 & -609 & -613 & -128 & -172 \\
268 & -4 & 1404 & -702 & -489 & 1198 \\
1491 & -1697 & 998 & -988 & -483 & -262 \\
610 & -943 & -164 & 157 & -91 & -19 \\
-247 & 1392 & -181 & 668 & 823 & -520 \\
-1834 & 722 & -1013 & 775 & 214 & 15
\end{array}\right]
$$

where the units for each matrix are $\mathrm{N} / \mathrm{m}$ and $\mathrm{N}$ sec/m, respectively. In both cases, the estimated stiffness matrix $\hat{\mathbf{K}}$, resembles closely what should be calculated by a finite element formulation based on the underlying physics of the problem. In other words, each inter-story stiffness is placed on the upper and lower diagonals as negative values except at the top story. Furthermore, the sum of inter-story stiffness of the adjacent stories is found along the diagonal except at the top story where it is simply the stiffness of the top story alone. The estimated stiffness matrices $\hat{\mathbf{K}}$, have significant values along the tri-diagonals with positive values along the diagonals and negative values along the off-diagonals. The off-diagonal values are close to the analytical value of story stiffness $\left(1.9 \times 10^{6} \mathrm{~N} / \mathrm{m}\right)$. Most of the off-diagonal stiffness terms fall within $5 \%$ of their theoretical values with slightly higher levels of error (approximately 20\%) for the stiffness values at the top level of 
the structure. The diagonal values are less than the sum of inter-story stiffness of the adjacent stories with values ranging from $15 \%$ to $24 \%$ less than that theoretically predicted. The errors are likely attributable to the lack of complete rigidity between the columns and each floor as assumed in our interpretation of the stiffness matrix.

It should be noted that there is a lack of symmetry in the stiffness matrices (Equations (50) and (52)) in addition to non-zero values found outside of the tri-diagonal terms. This can be attributed to the discrepancy between physics-based and data-driven models depicted in Figure 3(a) and (b). The roles of $\mathbf{B}$ in the two models differ. Furthermore, inclusion of $\mathbf{B}$ in the least square solution of the N4SID algorithm (Equation (14) in the companion paper [9]) will deteriorate the physical quality of A during the estimation stage leading to stiffness matrices that are not perfectly symmetric and populated with trivial but non-zero values off of the three main diagonals. This speculation is verified by the results of the numerical study (Section 4.2) where an identical set of system and input matrices ( $\mathbf{A}$ and $\mathbf{B}$, respectively) are used for the white-box and gray-box model. In that case, the stiffness matrix estimated (as shown in Table I) is perfect when the output measurements are noise-free.

Unfortunately, the damping matrices $\hat{\mathbf{C}}$ extracted from the input-output and output-only analyses do not correspond to any underlying physics of the problem. As previously studied in the numerical examples, damping estimation for lightly damped structures with relatively high signal-to-noise ratios is generally very challenging. As a result, the damage detection analyses conducted henceforth is confined only to the estimated stiffness matrix.

\subsection{Damage detection}

In this study, the location and severity of structural damage will be quantified by the stiffness change ratio $\left(S C R_{i j}\right)$, which is defined as follows:

$$
S C R_{i j}:=\left(k_{i j}^{D}-k_{i j}^{0}\right) / k_{i j}^{0}
$$

where $k_{i j}^{0}$ and $k_{i j}^{D}$ correspond to the $i$ th row, $j$ th column element of the estimated stiffness matrix $\hat{\mathbf{K}}$ for the baseline and damaged structure, respectively. Stiffness loss (i.e., damage) would correspond to a negative SCR for the diagonal terms $\left(S C R_{i i}<0\right)$ and a positive SCR for the superdiagonal $\left(S C R_{i}\right.$ $(i+1)>0)$ and subdiagonals $\left(S C R_{(i+1) i}>0\right)$. Figure 7 displays the SCR for the diagonal, superdiagonal, and subdiagonal terms of the stiffness matrix for the six damage cases using inputoutput data sets. As seen in Figure 7(a), the SCR of the diagonals closely match the damage introduced in the first and second stories. The SCR of floor 1 increases proportionally with the damage applied to the first story columns. Furthermore, the SCR of floor 2 in damage cases 5 and 6 also accurately depicts the applied damage to the second-story columns. However, slightly elevated

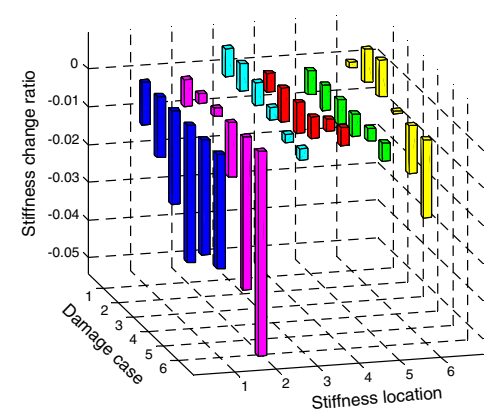

(a)

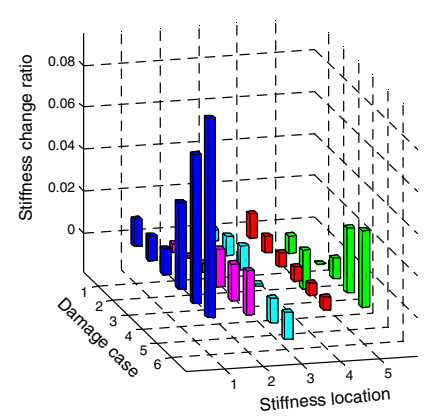

(b)

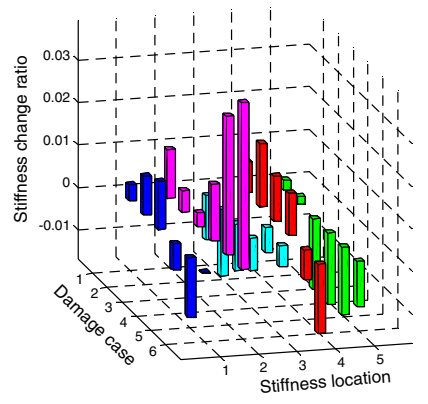

(c)

Figure 7. Stiffness change ratio (SCR) corresponding to the six damage cases: (a) diagonal $\left(S C R_{i i}\right)$; (b) superdiagonal $\left(S C R_{i(i+1)}\right)$; and (c) subdiagonal $\left(S C R_{(i+1) i}\right)$ terms in the stiffness matrix. 
SCRs are also found in other locations, especially on floor 6. In the case of the output-only analysis, similar damage detection results are confirmed up to a certain degree of damage. Figure 8 displays the SCR based on the output-only analysis. Generally, the SCR of the output-only stiffness matrices change more dramatically than those in the input-output case. Especially for relatively severe damage cases (e.g., damage case 5), large changes in the SCR is observed. In the case of damage case 6 ,which is the most severe damage scenario, output-only system identification by N4SID yields an unreliable model; as a result, damage detection is not conducted for this case. This implies that the input to the system is needed for precise quantitative detection of a wide range of damage states in a structure.

It should be noted that the location and severity of structural damage is identified using the relative stiffness change between the baseline and damaged structure. This implies that estimation of the system absolute stiffness is not an absolute requirement. As a result, the methodology does not require exact mass information for the purpose of damage detection. Specifically, the requirement for solving Equation (28) is that the mass ratio be known but not the absolute mass quantity.

\subsection{Damage detection with partial knowledge of the structure mass}

The estimation of the physical parameters of the system and subsequent damage detection is also conducted under the assumption of partial a priori knowledge of the structure's mass. For example, it is assumed that the first-floor mass is unknown; for the other floors, the story mass is assumed to be $862 \mathrm{~kg}$. Similar to the previous section, the gray-box model is utilized to extract the physical parameters of the system. By solving Equation (40), the spatial distribution of the stiffness $\mathbf{K}$, and damping $\mathbf{C}$, is calculated as follows:

$$
\hat{\mathbf{K}}=\left[\begin{array}{rrrrrr}
2,982,389 & -1,796,520 & 378,928 & -95,490 & 80,844 & -61,559 \\
-1,788,583 & 3,032,469 & -1,939,778 & 407,177 & 82,730 & -99,864 \\
439,303 & -1,827,917 & 2,932,826 & -1,892,131 & 534,577 & -131,916 \\
-145,435 & 507,923 & -2,002,127 & 3,187,329 & -1,870,920 & 299,167 \\
-33,299 & -73,415 & 440,842 & -1,907,798 & 2,969,031 & -1,462,394 \\
5980 & -116,316 & 130,940 & 298,632 & -1,645,762 & 1,343,867
\end{array}\right]
$$

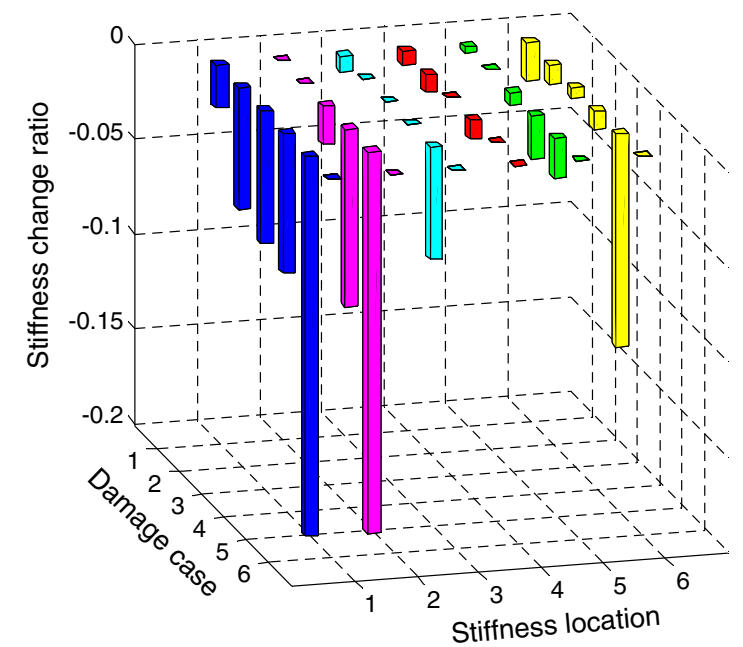

Figure 8. Stiffness change ratio (SCR) of the main diagonal terms of the stiffness matrix $\left(S C R_{i i}\right)$ for the output-only data sets. 


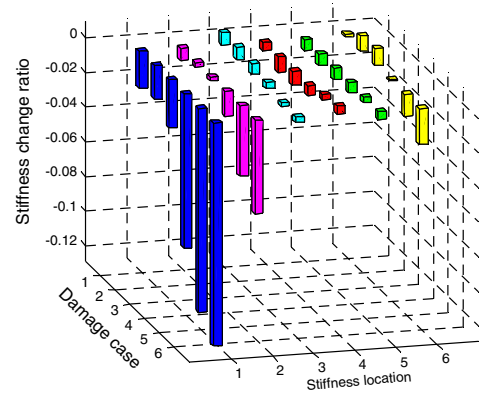

(a)

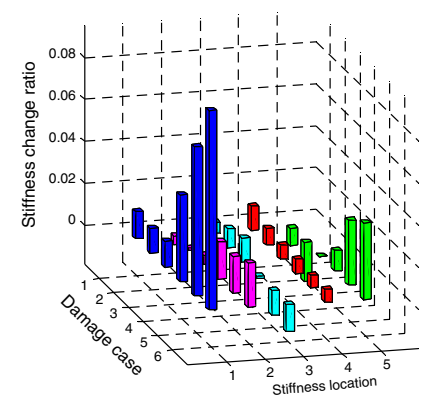

(b)

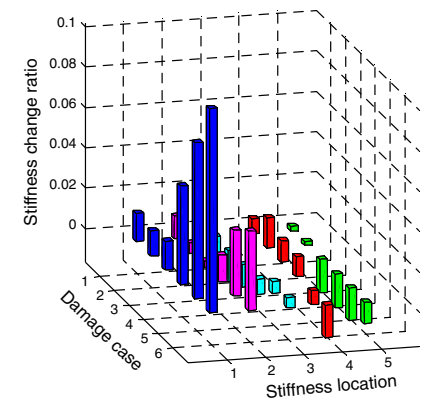

(c)

Figure 9. Stiffness change ratio (SCR) without knowledge of the first-floor mass for the following: (a) main diagonal $\left(S C R_{i i}\right)$; (b) superdiagonal $\left(S C R_{i(i+1)}\right)$; and (c) subdiagonal $\left(S C R_{(i+1) i}\right)$ stiffness matrix elements using the input-output data sets.

$$
\hat{\mathbf{C}}=\left[\begin{array}{rrrrrr}
1055 & -445 & 1059 & -572 & -54 & 183 \\
79 & -192 & 1659 & -995 & -917 & 555 \\
-398 & -292 & 1264 & -192 & -1721 & 195 \\
-429 & 175 & -610 & 783 & -1373 & -174 \\
-2 & 146 & -819 & 177 & 693 & -1088 \\
923 & -109 & -596 & 294 & 2469 & 832
\end{array}\right]
$$

Because of the absence of the first-floor mass, the stiffness and damping parameters associated with the first-floor mass have changed compared with Equations (50) and (51). Regardless, the SCR calculated for the six damage scenarios (Table III) and depicted in Figure 9 is still capable of reliably revealing the damage in the structure. Compared with Figure 7, which is based on complete a priori knowledge of the system mass, the SCR results of Figure 9 correlate more closely to the damage scenarios. This implies the following: (1) partial information on mass may still result in accurate estimation of the absolute stiffness; and (2) for the purpose of damage detection, comparable damage detection results are encountered with partial information on the structure mass.

\section{CONCLUSIONS}

In this paper, the estimation of the physical parameters of a structure (i.e., mass, stiffness, and damping) using input-output or output-only data sets was presented in detail. The framework considered two seemingly independent state-space models: a physics-based model (i.e., white-box model) and a data-driven mathematical model derived using the subspace system identification method (i.e., black-box model). Through observability canonical form conversion, the two models are shown to be closely related allowing for the derivation of a gray-box model from which structural parameters can be extracted. First, a numerical simulation study using a threestory-lumped mass shear structure was undertaken to illustrate the performance of the proposed system identification method. Outstanding results were observed for the estimation of the structure mass and stiffness parameters; estimation errors less than $1 \%$ were obtained even when noise in the sensor signals was high $(>3 \%)$. Next, an experimental study was undertaken with a single-bay steel frame structure mounted to a full-scale shake table for the application of base motion. For both input-output and output-only data sets, the stiffness of the structure was extracted. Using the SCR, the introduction of minor damage in the structure was easy to identify including the location and severity of the damage. Although the input-output data set gave the best results, the output-only data set also performed well for five of the six damage cases. Finally, absolute knowledge of the structural mass is not required to yield SCRs sufficiently sensitive to the damage introduced. It can 
be concluded that the proposed gray-box framework has shown great promise for structural health monitoring of civil engineering structures exposed to base motions such as earthquakes.

Future work is directed towards the establishment of the concept of data-driven finite element models. Unlike the conventional concept of finite element models, where an analytical approach is taken to discretization of the system based on physical principles, data-driven finite element models would realize discretized computational models based on system identification methods. Toward this aim, the gray-box approach proposed herein is extended to distributed mass flexural structural systems (i.e., beams, plates, etc.), which more closely depict real structural systems.

\section{ACKNOWLEDGMENTS}

The authors would like to gratefully acknowledge the generous support offered by the National Science Foundation under Grant CMMI-0726812 (Program Manager: Dr. S. C. Liu). The authors would also like to thank Prof. C. H. Loh of National Taiwan University and the NCREE staff for their generous support during the experimental testing at the NCREE shake table facility.

\section{REFERENCES}

1. Ljung L. System Identification: Theory for the User. Prentice Hall: Upper Saddle River, NJ, 1999.

2. Gevers M. A personal view on the development of system identification. 13th Federation of Automatic Control Symposium on System Identification (IFAC SYSID), Rotterdam, Netherlands, 2003.

3. Betti R. Time-Domain Identification of Structural Systems from Input-output Measurements. Springer: Vienna, Austria, 2008.

4. Hjelmstad KD, Banan MR, Banan MR. Time-domain parameter estimation algorithm for structures. I: computational aspects. ASCE Journal of Engineering Mechanics 1993; 121(3):424-434.

5. Larimore WE. Canonical variate analysis in identification, filtering, and adaptive control. 29th Conference on Decision and Control, Honolulu, HI, 1990.

6. Verhaegen M. Identification of the deterministic part of MIMO state space models given in innovations form from input-output data. Automatica 1994; 30(1):61-74.

7. Van Overschee P, De Moor B. N4SID: subsapce algorithms for the identification of combined deterministic-stochastic systems. Automatica 1994; 30(1):75-93.

8. Ljung L. System Identification Toolbox 7. The Mathworks, 2009.

9. Kim J, Lynch JP. Subspace system identification of support excited structures—part i: theory and black-box system identification. Earthquake Engineering and Structural Dynamics 2012 DOI: 10.1002/eqe.2184.

10. Kailath T. Linear Systems. Prentice-Hall: Englewood Cliffs, NJ, 1980.

11. Chen CT. Linear Systems Theory and Design. CBS College Publishing: New York, NY, 1984.

12. Xiao H, et al. An input/output-based procedure for fully evaluating and monitoring dynamic properties of structural systems via a subspace identification method. Journal of Sound and Vibration 2001; 246(4):601-623.

13. Lus H, De Angelis M, Betti R. A new approach for reduced order modeling of mechanical systems using vibration measurements. Journal of Applied Mechanics, ASME, 2003; 70:715-723.

14. Brewer JW. Kronecker products and matrix calculus in system theory. IEEE Transactions on Circuits and Systems 1978; 25(9):772-781.

15. Brogan WL. Modern Control Theory. Prentice Hall: Upper Saddle River, NJ, 1991. 